\title{
A Novel Design of Intelligent Energy- Efficient Drinking Water Dispensing Systems
}

\author{
YONATHAN ${ }^{\mathrm{a}, 1}$, Shuo-Yan CHOU ${ }^{\mathrm{a}}$, Ray-Guang $\mathrm{CHENG}^{\mathrm{b}}$ and Anindhita \\ DEWABHARATA $^{\mathrm{a}}$ \\ ${ }^{a}$ Department of Industrial Management, National Taiwan University of Science and \\ Technology, Taiwan \\ ${ }^{\mathrm{b}}$ Department of Electronic and Computer Engineering, National Taiwan University of \\ Science and Technology, Taiwan
}

\begin{abstract}
Energy efficiency and conservation have become a concern as many issues raised up regarding the increase in energy usage. Besides lighting and HVAC, a conventional drinking water dispenser also consumes plenty of huge electric energy in a building. However, most of them are wasted in vain as the machine will constantly repeat boiling and cooling the water even though no one uses the machine. In addition, the water that comes to the machine needs to boiled first to ensure it is safe to consume, then the water will be chilled and moves to warm-water tank and cold-water tank. Also, the machine needs to have sterilization which will heat all the water. Overall, the energy management in conventional drinking water dispenser is indigent. In this study, the design of intelligent energy-efficient drinking water dispensing system will be elaborated, including the architecture, key features, and analysis. Utilized by the Internet of Things and Cyber-Physical Systems, Intelligent Water Dispenser is able to maintain the energy efficiency.
\end{abstract}

Keywords. Internet of Things, Cyber-Physical Systems, Machine-to-Machine Communication, Energy Efficiency

\section{Introduction}

Drinking a water is essential for our health as it helps maintain the balance of body fluids. The human body is composed of about $60 \%$ water. The functions of these bodily fluids include digestion, absorption, circulation, the creation of saliva, transportation of nutrients, and maintenance of body temperature. Access to safe drinking-water is essential to health, a basic human right and a component of effective policy for health protection. Unfortunately, the tap water in Taiwan is safe for drinking only after boiling. As Taiwan is on a seismic active zone, there is a large number of earthquakes happen. It can damage the water delivery system allowing contaminants to enter the water prior to it reaching the tap [1]. Executive Yuan R.O.C. [2] stated that the tap water in Taiwan goes through multi-stage drinking-water processing in water treatment plant. Otherwise, it might contain impurities and microorganism which cannot be seen through naked eyes.

Water dispenser, which widely used in most public buildings in Taiwan, provides a

\footnotetext{
${ }^{1}$ Corresponding Author, Email m10501813@gapps.ntust.edu.tw.
} 
comprehensive way to fulfill the demand of drinking-water. The machine has a filtration function that can remove impurities and process the water to be ready to consume. In addition, the government also concern about the machine that the environmental authorities conduct an irregular inspection to ensure the safety of the drinking-water. People can fill up their bottles and drink the water without safety concerns. Surprisingly, the energy usage of the water dispenser takes a significant ratio in total energy usage in a building.

The current water dispenser, Union UN-9503AG-1, that is commonly used in Taiwan consumes almost 1.6 Kilowatt-hour $(\mathrm{kWh})$ in 24 hours, with the usage of 1,100 watts for boiling the water and 245 watts for chilling the water [3]. However, most of the energy usages in the water dispenser are wasted in vain as the machine will constantly repeat boiling and chilling the water even though no one uses the machine. In addition, the water that comes to the machine needs to boiled first to ensure it is safe to consume, then the water will chill down and moves to a warm-water tank and cold-water tank. In addition, the machine needs to have sterilization, which will boil all the water. Overall, the energy management in conventional drinking water dispenser is indigent. This is also contradictive with the fact that energy efficiency and energy conservation are blowing up as the current issue of the world to reduce the global warming effects.

As the water dispensers are widely used in public building in Taiwan and each building might have at least one water dispenser in a floor, this could be accounted as a huge wasted energy for the entire island. Different factors such as user behavior, dispenser's location, or weather might significantly have an impact on the total energy usage of the water dispenser. Thus, a specific design should be prepared for reaching the energy efficiency goal yet will not sacrifice the service level for the user as water is the basic human need.

In this study, a novel design will be created to enhance the usage of water dispensing system to be more efficient in terms of energy usage. Wide varieties of factors will be analyzed and will propose a new solution to tackle those issues. An innovative way is introduced to enhance the water dispensing system's usage into efficient usage and could adapt to its usage. Overall, the water drinking service will be the focus with both goals of energy efficiency and healthcare support for the users.

\section{Literature Review}

\subsection{Internet of Things}

The Internet of Things (IoT) is an emerging global internet-based information architecture facilitating the exchange of goods and services [4]. The IoT has the purpose of providing an IT-infrastructure facilitating the exchange of "things" in a secure and reliable manner. It enables physical objects to seamlessly integrated into the information network [5]. In the IoT paradigm, many of the objects that surround us will be on the network in one form or another. Radio Frequency Identification (RFID) and sensor network technologies will rise to meet this new challenge, in which information and communication systems are invisibly embedded in the environment around us [6]. This results in the generation of enormous amounts of data which have to be stored, processed and presented in a seamless, efficient, and easily interpretable form. 
Within the Internet of Things, IoT platforms are essentially software products, which offer comprehensive sets of application-independent functionalities that can be utilized to build IoT applications. The nature of individual IoT platforms therein may vary considerably as providers focus on different aspects of the IoT technology stack and correspondingly include diverse sets of functionalities in their offerings. Hence, there is no standard configuration of an IoT platform, but a multitude of IoT platforms exists, which address specific needs and areas of application.

From a technological point of view, the implementation of an IoT application requires the integration of a range of information and communication technologies in the form of hardware and software, as described earlier. Some of the most important challenges which IoT innovators are currently facing in this context relate to, e.g., device level energy supply, identification and addressing, Internet scalability, security, and personal privacy, as well as standardization and harmonization.

\subsection{Cyber-Physical Systems}

The term Cyber-Physical Systems (CPS) refers to a new generation of systems with integrated computational, networking, and physical capabilities that can interact with humans through many new modalities [7]. The ability to interact with, and expand the capabilities of, the physical world through computation, communication, and control is a key enabler for future technology developments. Opportunities and research challenges include the design and development of next-generation airplanes and space vehicles, hybrid gas-electric vehicles, fully autonomous urban driving, and prostheses that allow brain signals to control physical objects.

CPS will transform how humans interact with and control the physical world. Zeroenergy buildings and cities, extreme-yield agriculture, near-zero automotive fatalities, perpetual life assistants, location-independent access to medical care, situation-aware physical critical infrastructure, blackout-free electricity, and safe evacuation from hazardous areas are but some of the many societal benefits that CPS will deliver. CPS must operate dependably, safely, securely, efficiently and in real-time. CPS represent a confluence of technologies in embedded systems, distributed systems, dependable systems, real-time systems with advances in energy-efficient networking, microcontrollers, sensors, and actuators.

\subsection{Machine-to-Machine (M2M) Communication}

$\mathrm{M} 2 \mathrm{M}$ is about enabling the flow of data between machines and machines, and ultimately machines and people. M2M communications involve automated transfer of information and commands between two machines with no human intervention at either end of the system [8].

The characteristics of M2M networks are quite different from those of conventional wired or wireless networks [9]. M2M networks are composed of large numbers of nodes, since the main subject participating in M2M communication is a machine or object, or indeed it can be everything around us. To give such a large number of objects (millions or billions) the ability to communicate, the cost must be low [10]. Because most machines are battery operated, energy efficiency is the most important aspect. As the machine senses itself or its surrounding physical environment, the traffic per machine is 
very small. However, data are generated from a large number of objects, and because the data generation period, amount, and format are all different, a large quantity of data is generated. While M2M communication can occur without human intervention, operational stability and sustainability are also required.

\section{Fundamental Concept}

In this part, the fundamental concept of intelligent drinking water service systems will be elaborated. There will be an explanation about the current concept of water dispensing systems, design and requirements needed for applying the intelligent drinking dispensing systems, how it could reduce the energy usage, and how it can influence the user behavior toward better healthcare service.

\subsection{Concept of Drinking Water Dispensing Systems}

As the water dispenser serves three kinds of water (hot, warm, and cold), there will be three different tanks inside the machine. Each tank has the different capacity with the hot tank has the biggest capacity of 22 liters followed by warm tank and cold tank for 7.8 liters and 4 liters respectively. Firstly, the water comes through a pipe to the hot tank and then boiled there. After assuring the water boiled, then it will move and chill down to warm tank and cold tank sequentially. Each tank has insulation function to keep the water at their desired temperature. All water from each tank will come out into a single valve, which has a switch according to what the user selects at the moment. Figure 1 shows the water flow in the water dispenser.

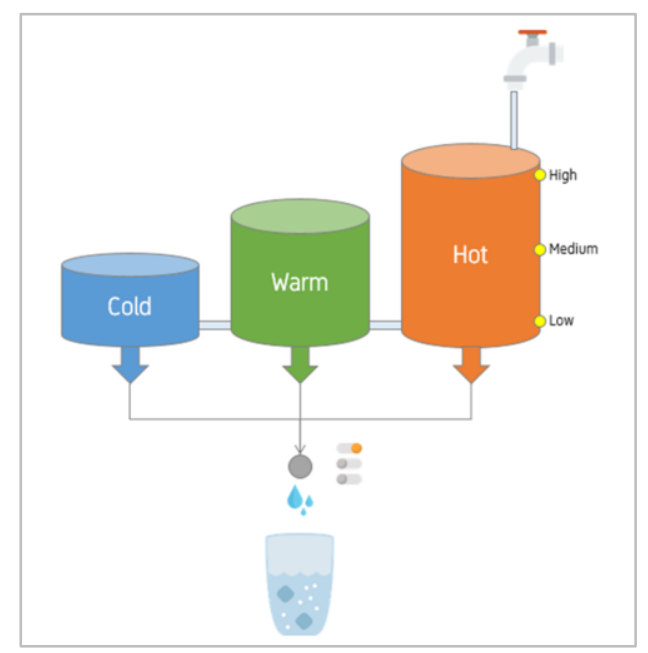

Figure 1. Concept illustration of the water dispenser tank.

The three tanks have its own temperature setting. The hot tank will keep the hot water from $50^{\circ} \mathrm{C}$ to $98^{\circ} \mathrm{C}$, while warm tank for $30^{\circ} \mathrm{C}$ to $50^{\circ} \mathrm{C}$, and the cold tank will keep the icy water from $10^{\circ} \mathrm{C}$ to $30^{\circ} \mathrm{C}$. 
When the water dispenser starts up for the first time, it takes time to boil the water first. It needs to boil for 10 minutes in $97^{\circ} \mathrm{C}$ to ensure the water safety. Afterward, the water will transfer to warm tank to chill down and then to cold tank to chill down as well. The length of water boiling depends on the current temperature of the water. If the current temperature is below $85^{\circ} \mathrm{C}$, then it will take 20 minutes to boil. If the temperature is at $90^{\circ} \mathrm{C}$ or $98^{\circ} \mathrm{C}$, then it will take 10 minutes and 120 seconds respectively to boil the water.

While the temperature in the hot tank is falling down, they need to boil again. When the hot water is $2^{\circ} \mathrm{C}$ below the lower bound, then it will boil again using both main heater and sub-heater until it reaches the upper bound. When it just $1^{\circ} \mathrm{C}$ below the lower bound, it will boil only using the main heater. This scheme will repeat over time based on the current temperature in the hot tank. It is not energy efficient as it will consume 1,100 watts to boil the water, moreover if the hot tank water level is set on high and the demand is not that high during the moment.

Each tank has an insulation function for the water inside the tank to ensure it is suitable for the temperature setting for each tank. While the temperature is not in the range of current setting, then the insulation will turn off and then either heater will start boiling the water or compressor will start to chill the water.

Sterilization is important to ensure the safety of the water, tanks, and valves inside the water dispenser. This function can be set up at a different time for each day. Normally, the machine will go to the sterilization at least once a week and mostly on a midnight. During the sterilization, the water dispenser cannot be interrupted and will not serve the water. Though it is important, sterilization actually consumes a huge of energy as it needs to boil all the water, tanks, and valves.

Actually, there is a power saving mode in the water dispenser which allows the dispenser to enter the sleep mode during the designated time. The user can adjust the sleep mode period for each day. However, this sleep mode can be interrupted easily by pressing the button in the water dispenser. When a machine in the power saving mode, the water charge will be ignored and will continue after the power saving period ends. The water also will not boil nor chill. However, when it comes to sterilization, the dispenser will proceed the sterilization first and then enter the power saving mode.

\subsection{IoT-Based Drinking Water Dispensing Systems}

The enormous increase in users of Internet and modifications on the internetworking technologies enable networking of everyday objects. "Internet of Things (IoT)" is all about physical items talking to each other, machine-to-machine communications and person-to-computer communications will be extended to "things". Based on the current drinking water dispensing system, IoT certainly could improve the effectiveness of the machine.

Several studies tried to develop similar things. Huang and Tsai [11] use a Micro Controller Unit program control in the circuit design to control the water release time and flow rate of the high-temperature boiled water and low-temperature boiled water and mix the high-temperature boiled water with the low-temperature boiled water to achieve the boiled water at the temperature and amount required by users. It can actually achieve the purposes of convenient and practical use and water saving. While Chen and $\mathrm{He}$ [12] use a single-chip microcomputer for their control processing unit and adapt fuzzy control as a control strategy to reduce ineffective heating of a drinking water dispenser. 
In this study, a series of sensors will be attached to the dispenser to catch real-time data, then it will be analyzed to decide the policy to the dispenser in order to reduce the energy consumption. Such modification, like when the water dispenser will heat up or how much water should chill down will be the resulting policy to the water dispenser. The modification of each dispenser will be different depends on its location and usage. For example, the water dispenser in the sports center might have a different pattern to the water dispenser in the office or any other places.

The growing number of sensors particularly plays a big role in the implementation of IoT-based drinking water dispensing system. Several sensors will be attached to the machine to catch real-time data that later will be analyzed, including water level, water flow, temperature, water valve, TDS, electric plug, outdoor weather, and schedule activities.

Decision making in the water dispenser scheme has to be based on accurate forecasts of the water demand. Therefore, water consumption forecasts are important tools in the intelligent drinking water service system. This study also extends to the predictive maintenance. The aim of predictive maintenance is first to predict when equipment failure might occur, and secondly, to prevent the occurrence of the failure by performing maintenance. Sleeping mode and sterilization time could also set dynamically. It is better to perform the sleeping mode and sterilization in their optimal time. Water dispenser can enter the sleeping mode when there is not much demand in a current period of time, moreover in the midnight. The system could easily set the start and end time for the sleeping mode to minimize the energy usage as optimal as possible. Seamlessly, water level and temperature could be predicted to the optimal number. The water level should be adjustable easily toward the upcoming demand.

\section{Framework Design}

In this section, the framework design of intelligent drinking water service systems, including system architecture and design model will be elaborated. Furthermore, the analysis and scenarios toward the goal of energy efficiency will be expanded here.

\subsection{System Architecture}

By using the Internet of Things to carry out the Cyber-Physical Systems, the water dispenser will be able to manipulate this usage and connect it to human. Several sensors will be attached to the machine and they will connect to the gateway. A single gateway is in charge of several water dispensers in a nearby location, e.g.: for a building or a complex. Afterward, all the data will be transferred to the cloud that connected to the Application Programming Interface (API). This API can serve to all platform that will be developed, including the website and mobile app. Figure 2 depicts the high-level systems architecture of the intelligent water dispenser as explained above.

Based on this architecture, each dispenser will be able to connect with other dispensers since they are in the same gateway. The outdoor sensor for weather and schedule will be connected directly to the API. All the data will be analyzed in the platform and the result will be shown in the monitoring and control apps. 


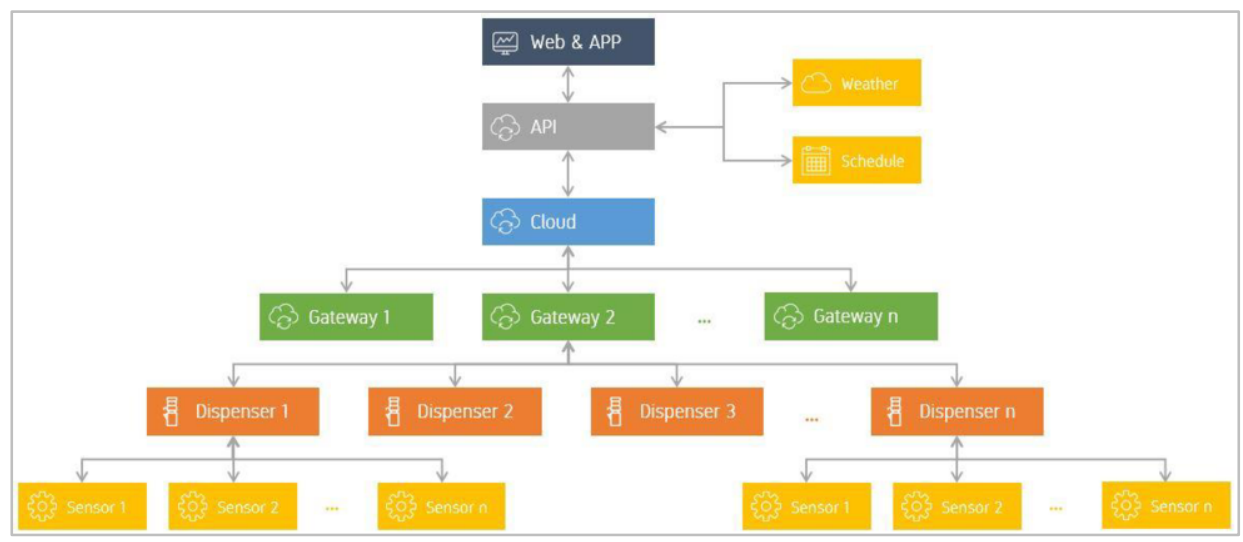

Figure 2. Systems architecture of the intelligent water dispenser.

\subsection{Intelligent Drinking Water Dispensing Systems}

In this study, several framework designs will be created for achieving the goal of energyefficient water dispensing system. Based on the preliminary study and collected data, there will be four major framework design: user behavior analytics, predictive analytics, machine to machine communication, and predictive maintenance. Each of framework design will be elaborated in this section.

\subsubsection{User Behavior Analytics}

The important part of the water dispensing system is the user behavior. Each dispenser will have different user behavior pattern according to its location. It is important to analyze the user behavior toward their water intake. For example, the office water dispenser will have a normal operating scheme during the work hour, the water dispenser in sports center might have a higher volume of cold water or the water dispenser in the home doesn't need to boil and chill during the work hour since no one uses it.

The relationship between user behavior and the location of the water dispenser will affect the water dispenser scheme. If the water dispenser can acknowledge the user behavior, then it will be a better system as it can cut off the unnecessary boiling or chilling process. In addition, it could provide a better service level for the user.

This feature also enables the user to monitor their behavior and the water dispenser status. Figure 3 shows the monitoring page for a water dispenser status. Through the monitoring page, the user can see the status of the dispenser, including the temperature of each tank, water consumption, filter status, and energy usage.

\subsubsection{Predictive Analytics}

As the sensor will catch the real-time data continuously for the water consumption and energy usage, then the system could easily predict the upcoming demand. The user is able to see the historical usage and see the report about it.

Predictive analytics encompasses a variety of statistical techniques from data mining, predictive modeling, and machine learning, and that analyze current and 


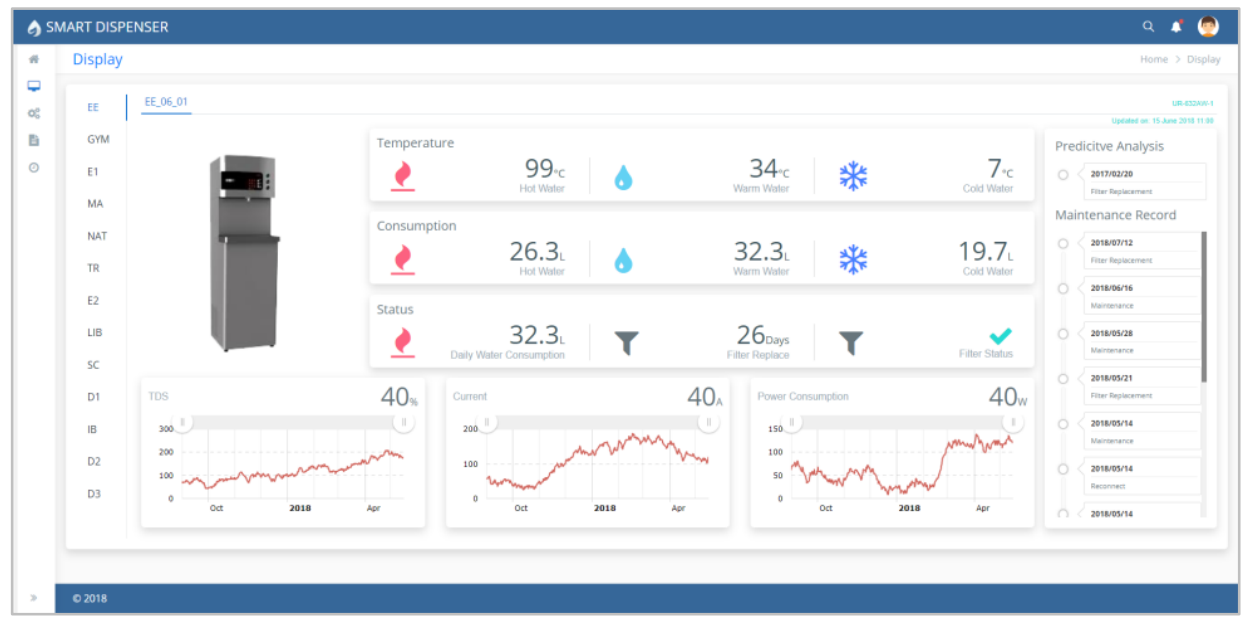

Figure 3. Monitoring page of the intelligent water dispensing systems.

historical facts to make predictions about future events. The system could predict the upcoming water consumption and water dispenser could react correctly to this demand. For the water consumption prediction, factors such as previous historical data on the same day, outdoor temperature, outdoor humidity, and time will affect the prediction. The prediction might not be accurate, but it can interpret a right direction towards future demand. Choosing the best algorithm and correct forecasting method will be another challenge to this predictive analytics. Expose the historical data might have a better insight into the water dispenser's demand pattern.

\subsubsection{M2M Communication}

Water dispenser can work in coordination since they know each other status. A set of rules can be built under the semantic rules for enabling the communication. The arrangement of the water dispenser will be optimal and can further reduce the energy consumption.

Based on historical data and predicted demand, the water dispenser could adapt correctly and work effectively regarding their boiling, chilling, and amount of water preserved in the tank. But, it just works in a single dispenser. By M2M, it will be more sophisticated as the dispenser can work in coordination. The multi dispenser will run the combined scheme, e.g.: the water dispenser in the first floor of the building can skip boiling the water since the dispenser knows that the second floor's dispenser has sufficient amount of hot water to cope the upcoming demand.

These connected water dispensers will empower a wide range of highly automated processes and cut off unnecessary cycle of the water dispenser. The key decision-makers will lead to the energy-efficient drinking water dispensing system.

\subsubsection{Predictive Maintenance}

Through attached sensors, the system will be able to gather supporting data. It will support maintenance that directly monitors the condition and performance of water dispenser during normal operation to reduce the likelihood of failures. It attempts to keep 
costs low by reducing the frequency of maintenance tasks, reducing unplanned breakdowns and eliminating unnecessary preventive maintenance.

Furthermore, with the historical data, the system could easily detect anomaly through the dataset. The core idea of predictive maintenance is to predict when equipment failure might occur based on condition-monitoring data. It will monitor the condition and performance of equipment during normal operation and thus reduces disruption of everyday operations. It could give early notification regarding the maintenance, machine breakdown, or filter change.

\subsection{Analysis}

The energy-efficient intelligent drinking water dispensing systems will utilize IoT as the main drive. Sensors enable systems to catch the real-time data and data analytic tools support the decision making of the system in order to perform effectively. Based on key features explained before, the systems should be able to further reduce the energy usage and work effectively. The whole system has different stages of innovation where each stage contributes prevalently to the study objectives.

\subsubsection{Intelligent learning}

The water dispenser has an ability to learn about the user behavior. Some machine learning technique, such as neural network or deep learning could be used to modeling each water dispenser. The systems will able to analyze how the dispenser is being used through the different cycle, different usage behavior, and different location. The sensors enable to capture the real-time data, while the software is responsible to make a decisionmaking system. Each dispenser will have a different scheme depends on its usage. It will also lead to creating an autonomous intelligent water dispensing systems.

\subsubsection{Adaptation}

An adaptation for reducing energy consumption is important to the water dispenser. The machine should able to adapt to different users. The boiling and chilling schedule will be more complex according to the current usage and predicted demand. This scheme will be able to reduce the energy consumption. Moreover, the systems will be able to predict the sleeping mode and sterilization schedule into the optimal schedule when it will not sacrifice the service level to the user and will not consume significant energy usage. The water dispenser will have a dynamic policy regarding their water processing policy. This enables the system to continuously observe the product. The change of product design is also a solution to optimize the usage, e.g.: size of the tank, water level in each tank.

\subsubsection{Connected products}

$\mathrm{M} 2 \mathrm{M}$ enables the water dispenser to be connected products. Through the information exchange and automation, the water dispenser will be able to work in coordination. There is the possibility of systems monitoring themselves and automatically responding to changes in the environment without human assistance. It will reduce unnecessary cycle during the dispenser process and thus reduce the energy consumption. Connected products allowing the water dispenser to be a service system where the machine should 
complement each other. It is also lead to a virtual distributed of the water dispenser.

\section{Discussion}

In this study, an IoT-based intelligent drinking water dispensing system is designed in order to reach the energy-efficient environment. This also will lead to reaching the effective and optimal usage of the water dispenser. The key elements that are incorporated into this system are the Internet of Things, Machine-to-Machine communication, and persuasive technology.

The intelligent water dispenser will contribute through its predictive analytics, user behavior analytics, and predictive maintenance. Moreover, it is also able to perform machine-to-machine communication that enables the machine to work in coordination. By having enough supply for upcoming demand in a certain location, intelligent water dispenser will satisfy the service level for the user. Besides, it also conserves more energy by cutting down several scheme patterns of water dispenser when it is unproductive.

Finally, the framework design of intelligent drinking water dispensing systems are generated. The designated web app to support the system are developed. Further analysis is also conducted to ensure it reaches the aim of this study. The proposed strategies are distinguished into four main parts, which are intelligent learning, adaptation, and connected products.

\section{References}

[1] A. B. Waters. (2013, May 13, 2018). Drinking Water - Taiwan. Available: http://www.arcticbluewaters.com/news-article/528244ff77972/Drinking-Water-Taiwan

[2] E. Y. R. O. C. T. Environmental Protection Administration, Drinking Water FAQs, ed. Taipei, 2012.

[3] E. Bureau. (2018, May 7, 2018). 冰 溫 熱型 飲 水 機. Available: http://www.energylabel.org.tw/purchasing/psearch/item.aspx?itemp $0=14373 \& \mathrm{p} 0=14 \&$ uid $=0 \&$ con $=1 \&$ cid $=14 \& \operatorname{cid} 2=6 \&$ year $=\&$ month $=\&$ day $=\&$ key $=\& \operatorname{cid} 1=0$

[4] R. H. Weber and R. Weber, Internet of things. Springer, Berlin Heidelberg, 2010.

[5] B. Fabian, Secure name services for the Internet of Things, PhD thesis, TU Berlin, 2008.

[6] J. Gubbi, R. Buyya, S. Marusic, and M. Palaniswami, Internet of Things (IoT): A vision, architectural elements, and future directions, Future generation computer systems, vol. 29, no. 7, pp. 1645-1660, 2013.

[7] R. Baheti and H. Gill, Cyber-physical systems, The impact of control technology, vol. 12, no. 1, pp. 161$166,2011$.

[8] J. Holler, V. Tsiatsis, C. Mulligan, S. Karnouskos and D. Boyle, From Machine-to-machine to the Internet of Things: Introduction to a New Age of Intelligence. Academic Press, Boston, 2014.

[9] ETSI TS 102 691, Machine-to-Machine communications (M2M); Smart Metering Use Cases, V1.1.1, 2010.

[10] J. Kim, J. Lee, J. Kim and J. Yun, "M2M Service Platforms: Survey, Issues, and Enabling Technologies," IEEE Communications Surveys and Tutorials, vol. 16, no. 1, pp. 61-76, 2014.

[11] C. J. Huang and F. T. Tsai, Research and development of a Practical Water Dispenser, Applied System Innovation (ICASI), 2017 International Conference on, 2017, pp. 1225-1228: IEEE.

[12] Z. Chen and Y. He, A smart power saver based on composite switch and self-learning fuzzy control for drinking water dispenser, Power and Renewable Energy (ICPRE), IEEE International Conference on Power and, 2016, pp. 275-278: IEEE. 